\title{
The $s \bar{s}$ component of the proton and the strangeness magnetic moment
}

\author{
B. S. Zou* \\ Institute of High Energy Physics, CAS, P.O.Box 918, Beijing 100049, China \\ D. O. Riskat \\ Helsinki Institute of Physics and Department of Physical Sciences, POB 64, 00014 University of Helsinki, Finland
}

(Dated: November 6, 2018)

\begin{abstract}
A complete analysis is given of the implications of the empirical indications for a positive strangeness magnetic moment $\mu_{s}$ of the proton on the possible configurations of the uuds $\bar{s}$ component of the proton. A positive value for $\mu_{s}$ is obtained in the $s \bar{s}$ configuration where the uuds subsystem is in an orbitally excited state with $[4]_{F S}[22]_{F}[22]_{S}$ flavor-spin symmetry, which is likely to have the lowest energy. The configurations in which the $\bar{s}$ is orbitally excited, which include the conventional $K^{+} \Lambda^{0}$ configuration, with exception of that in which the uuds component has spin 2, yield negative values for $\mu_{s}$. The hidden strangeness analogues of recently proposed quark cluster models for the $\theta^{+}$pentaquark give differing signs for $\mu_{s}$.
\end{abstract}

Three recent experiments on parity violation in electron- proton scattering suggest that the strangeness magnetic moment of the proton $\mu_{s}$ is positive [1, 2, 3]. In contrast theoretical calculations have led to negative values for this observable, with but few exceptions [4, 5, 6, 7, 8, 9]. Here the implications of the empirical result for the configuration of the constituents of the proton is considered by a calculation of $\mu_{s}$ for all positive parity configurations of the $u u d s \bar{s}$ system with one constituent in the first orbitally excited state, which may be contained in the proton. The results are given in the form of simple general expressions, in which $\mu_{s}$ is proportional to the $s \bar{s}$ probability of the configuration.

It is shown that $\mu_{s}$ is positive in the $u u d s \bar{s}$ configuration, which is likely to have the lowest energy, where the $\bar{s}$ quark is in the ground state and the uuds system is in the $P$-state. If in contrast the strange antiquark is in the $P$-state and the 4 quarks are in their ground state the strangeness magnetic moment is negative (except for the energetically unfavored case where the total spin of the uuds state equals 2). These configurations correspond to that of a fluctuation of the proton into a kaon and a strange hyperon, which is well known to lead to a negative value for the strangeness magnetic moment 10, 11, 12, 13].

Several intriguing configurations 14, 15, 16] for the system of 4 light flavor quarks and a strange antiquarks have been proposed to explain the structure of the (tentative) $\theta^{+}$pentaquark. Below their hidden strangeness analogues are considered from the point of view of the strangeness magnetic moment. While the diquarkdiquark configurations suggested in refs. 14, 15] with the $\bar{s}$ quark in its ground state yield positive values, the diquark-triquark configuration suggested in ref. [16] yields a negative value for the strangeness magnetic moment of the proton.
If the uuds quarks are in their ground state, the spatial state is completely symmetric $[4]_{X}$, and the Pauli principle requires that their flavor-spin state has the mixed symmetry $[31]_{F S}$ so as to combine with the color state $[211]_{C}$ to form the required total antisymmetry [1111]. The different flavor and spin state symmetry configurations that can combine to the required $[31]_{F S}$ mixed symmetry combination are listed in Table 1 [17]. The requirement of positive parity requires that for these configurations the strange antiquark has to be in the $P$-state.

If in contrast the strange antiquark $\bar{s}$ is in its ground state, the uuds system has to be in the $P$-state in order that the combination have positive parity. In this case the symmetry of the spatial state of the 4-quark system is reduced to $[31]_{X}$. When combined with the mixed symmetry color state $[211]_{C}$ this allows the flavor-spin combination to either be completely symmetric $[4]_{F S}$ or to have any one of the mixed symmetries $[31]_{F S},[22]_{F S}$ or $[211]_{F S}$. The combinations of flavor and spin symmetry configurations that lead to these symmetries are also listed in Table 【 17.

In the quark model the strangeness magnetic moment is defined as the matrix element of the operator

$$
\vec{\mu}_{s}=e \sum_{i} \frac{\hat{S}_{i}}{2 m_{s}}\left(\vec{l}_{i}+\vec{\sigma}_{i}\right),
$$

where $\hat{S}$ is the strangeness counting operator, with eigenvalue +1 for $s$ and -1 for $\bar{s}$ quarks and $m_{s}$ is the constituent mass of the strange quark.

Consider first the configurations, in which the antiquark is in the $P$-state. For these the spatial state of the uuds is completely symmetric $[4]_{X}$ with total orbital angular momentum 0 . The corresponding wave functions for a proton with spin up may then be written in the general form: 


$$
\begin{aligned}
& \psi=A_{s \bar{s}} \sum_{a, b, c} \sum_{m, s, M, J, j}(1,1 / 2, m, s \mid J, j) \\
& (S, J, M, j \mid 1 / 2,1 / 2) C_{[211] a,[31] a}^{[1111]} C_{[F] b,[S] c}^{[31] a}[211]_{C}(a) \\
& {[F](b)[S]_{M}(c) \bar{Y}_{1 m} \bar{\chi}_{s} \varphi\left(\left\{r_{i}\right\}\right) .}
\end{aligned}
$$

Here $[211]_{C},[F]$ and $[S]$ are the color, flavor and spin states denoted by their Young patterns respectively, and the sums over $a, b$ and $c$ run over the configurations of the [31][F][S] representations of $S_{4}$ for which the corresponding Clebsch-Gordan $C_{b, c}^{a}$ are non-vanishing [19]. The orbital and spin states of the $\bar{s}$ are denoted by $\bar{Y}_{1 m}$ and $\bar{\chi}_{s}$ respectively. $A_{s \bar{s}}$ is the amplitude of the $s \bar{s}$ component and $\varphi\left(\left\{r_{i}\right\}\right)$ is a symmetric function of the coordinates of the $u u d s \bar{s}$ system.

The configurations in the left column in Table \may be grouped according to their spin symmetries $[22]_{S},[31]_{S}$ and $[4]_{S}$. The uppermost configuration in the left column in Table【 which has the spin symmetry $[22]_{S}$ is expected to have the lowest energy if the uuds is in its ground state. This is the case both if the hyperfine interaction between the quarks is described by the color magnetic

TABLE I: Flavor and spin state symmetry configurations of the uuds quark states in the ground state and first orbitally excited $P$ state. The states are ordered from above after increasing matrix elements of the Casimir operator $-\sum_{i<j} \vec{\lambda}_{i} \cdot \vec{\lambda}_{j} \vec{\sigma}_{i} \cdot \vec{\sigma}_{j}$, where $\lambda_{i}$ are the $S U(3)_{F}$ generators. These matrix elements are listed in the brackets [17].

\begin{tabular}{ll|ll} 
uuds ground state & uuds $P$-state & \\
\hline$[31]_{F S}[211]_{F}[22]_{S}$ & $(-16)$ & {$[4]_{F S}[22]_{F}[22]_{S}$} & $(-28)$ \\
{$[31]_{F S}[211]_{F}[31]_{S}$} & $(-40 / 3)$ & {$[4]_{F S}[31]_{F}[31]_{S}$} & $(-64 / 3)$ \\
{$[31]_{F S}[22]_{F}[31]_{S}$} & $(-28 / 3)$ & {$[31]_{F S}[211]_{F}[22]_{S}$} & $(-16)$ \\
{$[31]_{F S}[31]_{F}[22]_{S}$} & $(-8)$ & {$[31]_{F S}[211]_{F}[31]_{S}$} & $(-40 / 3)$ \\
{$[31]_{F S}[31]_{F}[31]_{S}$} & $(-16 / 3)$ & {$[31]_{F S}[22]_{F}[31]_{S}$} & $(-28 / 3)$ \\
{$[31]_{F S}[31]_{F}[4]_{S}$} & $(0)$ & {$[31]_{F S}[31]_{F}[22]_{S}$} & $(-8)$ \\
{$[31]_{F S}[4]_{F}[31]_{S}$} & $(+8 / 3)$ & {$[4]_{F S}[4]_{F}[4]_{S}$} & $(-8)$ \\
& & {$[22]_{F S}[211]_{F}[31]_{S}$} & $(-16 / 3)$ \\
& {$[31]_{F S}[31]_{F}[31]_{S}$} & $(-16 / 3)$ \\
& {$[22]_{F S}[22]_{F}[22]_{S}$} & $(4)$ \\
& {$[211]_{F S}[211]_{F}[22]_{S}$} & $(0)$ \\
& {$[31]_{F S}[31]_{F}[4]_{S}$} & $(0)$ \\
& {$[211]_{F S}[211]_{F}[31]_{S}$} & $(8 / 3)$ \\
& {$[22]_{F S}[31]_{F}[31]_{S}$} & $(8 / 3)$ \\
& {$[31]_{F S}[4]_{F}[31]_{S}$} & $(8 / 3)$ \\
& {$[22]_{F S}[22]_{F}[4]_{S}$} & $(4)$ \\
& {$[211]_{F S}[22]_{F}[31]_{S}$} & $(20 / 3)$ \\
& {$[211]_{F S}[211]_{F}[4]_{S}$} & $(8)$ \\
& {$[211]_{F S}[31]_{F}[22]_{S}$} & $(8)$ \\
& {$[22]_{F S}[4]_{F}[22]_{S}$} & $(8)$ \\
& {$[211]_{F S}[31]_{F}[31]_{S}$} & $(32 / 3)$ \\
\hline
\end{tabular}

interaction or by the flavor and spin dependent hyperfine interaction $-C \sum_{i<j} \vec{\lambda}_{i} \cdot \vec{\lambda}_{j} \vec{\sigma}_{i} \cdot \vec{\sigma}_{j}$, where $C$ is a constant with the value $\sim 20-30 \mathrm{MeV}$, and which leads to the empirical ordering of the baryon resonances 17, 18]. For the states with this spin symmetry the total spin of the uuds system is $S=0$. The corresponding strangeness magnetic moment is negative:

$$
\mu_{s}=-\frac{1}{3} \frac{m_{p}}{m_{s}} P_{s \bar{s}},
$$

(in units of nuclear magnetons $\mu_{N}=e / 2 m_{p}$ ). Here $P_{s \bar{s}}=$ $A_{s \bar{s}}^{2}$ is the probability of the $s \bar{s}$ component and $m_{s}$ is the constituent mass of the strange quark.

The following two configurations in the left column in Table \ have the spin symmetry $[31]_{S}$ and thus $S=1$. For these configurations and all other configurations in the left column that have the same spin symmetry $\mu_{s}$ may be expressed in the general form:

$$
\mu_{s}=-\frac{m_{p}}{m_{s}}\left(\frac{7}{6}-\frac{1}{2} \bar{\sigma}\right) P_{s \bar{s}} .
$$

Here $\bar{\sigma}$ is the average of $\sigma_{z}$ of the $s$ quark in the configuration for $\mathrm{S}=1$ with z-projection $\mathrm{M}=1$. As $|\bar{\sigma}| \leq 1$, all of these configurations yield a negative strangeness magnetic moment in apparent conflict with the positive empirical value. The numerical values of $\bar{\sigma}$ are very similar - ie $41 / 72$ and $1 / 2$ - in the configurations with flavor-spin symmetry $[211]_{F}[31]_{S}$ and $[22]_{F}[31]_{S}$.

This result that the strangeness magnetic moment is negative if the $\bar{s}$ quark is in the $P$-state generalizes to all other configurations in the left column of Table $\square$ except for the energetically unfavored $[31]_{F S}[31]_{F}[4]_{S}$ configuration in which the uuds system has total spin 2. For this configuration $\mu_{s}$ takes the value:

$$
\mu_{s}=\frac{7}{6} \frac{m_{p}}{m_{s}} P_{s \bar{s}} .
$$

These results complete the analysis of the configurations with the $\bar{s}$ quark in the $P$-state.

In the configurations $\bar{s}$ quark is in its ground state, positive parity demands that the uuds system has to be in the $L=1$ state with mixed spatial symmetry $[31]_{X}$. In order to construct the corresponding spatial wave functions one may exploit a representation in terms of the following relative coordinates:

$$
\begin{aligned}
& \vec{\xi}_{1}=\frac{1}{\sqrt{2}}\left(\vec{r}_{1}-\vec{r}_{2}\right), \quad \vec{\xi}_{2}=\frac{1}{\sqrt{6}}\left(\vec{r}_{1}+\vec{r}_{2}-2 \vec{r}_{3}\right), \\
& \vec{\xi}_{3}=\frac{1}{\sqrt{12}}\left(\vec{r}_{1}+\vec{r}_{2}+\vec{r}_{3}-3 \vec{r}_{4}\right), \\
& \vec{\xi}_{4}=\frac{1}{\sqrt{20}}\left(\vec{r}_{1}+\vec{r}_{2}+\vec{r}_{3}+\vec{r}_{4}-4 \vec{r}_{5}\right),
\end{aligned}
$$

which when complemented with the center-of-mass coordinate form a complete set of basis vectors.

For these states the wave functions for the configurations in the right columns in Table \may for a proton 
with spin up then be written in the general form:

$$
\begin{aligned}
& \psi=A_{s \bar{s}} \sum_{a, b, c, d, e} \sum_{m, s, M, j}(1, S, m, M \mid J, j) \\
& (J, 1 / 2, j, s \mid 1 / 2,1 / 2) C_{[211] a,[31] a}^{[1111]} C_{[31] b,[F S] c}^{[31] a} C_{[F] d,[S] e}^{[F S] c} \\
& {[211]_{C}(a)[31]_{X, m}(b)[F](d)[S]_{M}(e) \bar{\chi}_{s} \varphi\left(\left\{r_{i}\right\}\right) .}
\end{aligned}
$$

Here $J$ is the total angular momentum of the uuds system. The three components of the spatial state may be described as normalized combinations of a spatially symmetric function that is multiplied by the unit vectors $\hat{\xi}_{1}, \hat{\xi}_{2}$ and $\hat{\xi}_{3}$.

The contribution to $\mu_{s}$ from all configurations in the right column of Table【 which have the spatial symmetry $[31]_{X}$ and spin symmetry $[22]_{S}$ are positive:

$$
\mu_{s}=\frac{m_{p}}{m_{s}}\left(\frac{1}{3}+\frac{2}{3} \bar{\ell}\right) P_{s \bar{s}} .
$$

Here $\bar{\ell}$ is the average value of the $z$-components of the orbital angular momentum of the $s$ quark in the uuds system for $m=1$ ( $M=0$ since $S=0$ for these configurations). These configurations thus give positive values for $\mu_{s}$ as $|\bar{\ell}| \leq 1 / 2$.

For the uppermost and energetically most likely configuration in the right column in Table \ $\bar{\ell}=1 / 4$ and therefore $\mu_{s}$ is simply:

$$
\mu_{s}=\frac{m_{p}}{2 m_{s}} P_{s \bar{s}} .
$$

The following symmetry configuration in the right column Table \ has both spatial and spin symmetry [31]. The contribution to $\mu_{s}$ may for all states with both spatial and spin symmetry [31] be expressed as:

$$
\begin{aligned}
& \mu_{s}=-\frac{m_{p}}{m_{s}} P_{s \bar{s}}, \quad(J=0), \\
& \mu_{s}=\frac{m_{p}}{m_{s}}\left(\frac{1}{3}+\frac{1}{3} \bar{\ell}+\frac{1}{3} \bar{\sigma}\right) P_{s \bar{s}}, \quad(J=1) .
\end{aligned}
$$

Of these configurations that with lowest energy has flavor-spin symmetry $[4]_{F S}[31]_{F}[31]_{S}$. In this configuration when $J=1 \bar{\ell}=1 / 4$ and $\bar{\sigma}=-1 / 18$.

¿From (7) one may finally infer that the contribution to $\mu_{s}$ from all the configurations in the right column of Table【 which have the spatial symmetry $[31]_{X}$ and spin symmetry $[4]_{S}$ are likewise positive and may be expressed in the form:

$$
\mu_{s}=\frac{m_{p}}{m_{s}}\left(\frac{5}{6}-\frac{1}{3} \bar{\ell}\right) P_{s \bar{s}} .
$$

Here the first term is the sum of the contributions of the $s$ and $\bar{s}$ quarks, which are $1 / 2$ and $1 / 3$ respectively. In the lowest of these configurations, which also has flavor symmetry $[4]_{F}, \bar{\ell}=1 / 4$. In the following one in order of increasing energy the flavor symmetry is $[31]_{F}$ and $\bar{\ell}=$ $19 / 54$. This completes the analysis of the configurations in the right column of Table \
These results reveal that $\mu_{s}$ is positive in the lowest lying 5-quark configurations, in which the strange antiquark $\bar{s}$ is in the ground state. For the lowest configuration $\mu_{s}=0.5 m_{p} / m_{s}$ times the probability $P_{s \bar{s}}$ for the preformed $s \bar{s}$ component in the proton. This result then leads to $P_{s \bar{s}} \sim 2 m_{s} / m_{p} \mu_{s}^{e x p} \sim \mu_{s}^{e x p}$. For $\mu_{s}^{e x p}$ the result of the SAMPLE experiment [1] is $\mu_{s}=$ $0.37 \pm 0.2 \pm 0.26 \pm 0.07$.

In order to explain the structure of the (tentative) $\theta^{+}(u u d d \bar{s})$ pentaquark, several clustered quark models have been proposed [14, 15, 16]. These quark cluster configurations have implications also for the pentaquark components with hidden strangeness in the nucleons. The magnetic moments of the pentaquark states in the cluster models have been calculated in ref. 20] for the exotic flavor antidecuplet and normal flavor octet baryons.

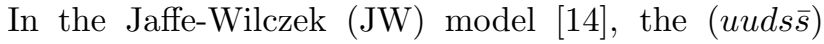
component in the proton should have the configuration $([u d][u s] \bar{s})$ with two scalar diquarks, $[u d]$ and $[u s]$, in relative $P$-wave, and the $\bar{s}$ quark in its ground state. The corresponding strangeness magnetic moment is:

$$
\mu_{s}=\frac{m_{p}}{3 m_{s}}\left(1+\frac{2 m_{s}}{m_{u d}+m_{u s}}\right) P_{s \bar{s}},
$$

where $m_{u d}$ and $m_{u s}$ are diquark masses for $[u d]$ and [us], respectively.

In the Shuryak-Zahed (SZ) 15] model, the $(u u d s \bar{s})$ is composed of one scalar diquark, one tensor diquark and the $\bar{s}$ antiquark. It has the same flavor configuration as in JW model. But the orbital excitation $\ell=1$ is now between the two quarks inside the tensor diquark instead of between two diquarks as in JW model. The two quarks in the tensor diquark have total spin $S=1$, which in combination with their relative $P$ - wave leads to total angular momentum $J=1$ of the tensor diquark. In this configuration $\mu_{s}$ is found to be:

$$
\mu_{s}=\frac{m_{p}}{2 m_{s}}\left(1+\frac{m_{q}}{3 m_{u s}}\right) P_{s \bar{s}} .
$$

Finally in the Karliner-Lipkin (KL) model [16], the $\theta^{+}$pentaquark is composed of a scalar $[u d]$ diquark and a $\{u d \bar{s}\}$ triquark with one orbital angular momentum $L=1$ between the diquark and the triquark. The total spin of the triquark is one half with a symmetric spin wave function for the two quarks.

An analogous configuration for the (uuds $\bar{s})$ component in the proton is $[u d]\{u s \bar{s}\}$ with $L=1$ between scalar diquark $[u d]$ and the spin- $1 / 2$ triquark $\{u d \bar{s}\}$. For this configuration $\mu_{s}$ is:

$$
\mu_{s}=-\frac{1}{3} \frac{m_{p}}{m_{s}} P_{s \bar{s}},
$$

which is negative definite and the same as for the $K^{+} \Lambda^{0}$ configuration. Another analogous configuration: [us]uds gives a positive $\mu_{s}$, but with a magnitude about 100 times smaller than that of given $[u s] u d \bar{s}$. A combination of these two configurations in the flavor SU(3) symmetry [19] still gives a negative definite value for $\mu_{s}$. 
These results for the quark cluster models, reveal that the $u u d s \bar{s}$ configurations in JW and SZ models give similar and positive values for $\mu_{s}$, while the configuration in KL model gives a negative value. A common feature for the JW and SZ models is that in both of these the $\bar{s}$ quark in its ground state and the uuds system has total angular momentum 1. The configurations in KL and the $K^{+} \Lambda^{0}$ meson cloud model lack that feature.

This analysis of the $(u u d s \bar{s})$ configurations in the shows that the lowest configurations with the $\bar{s}$ in the ground state give positive values for $\mu_{s}$, while the lowest configurations with the $\bar{s}$ in the $P$-state give negative values. The empirical indications [1, 2, 3] for a positive $\mu_{s}$ implies that the $\bar{s}$ is in the ground state and that the uuds system is orbitally excited. This is at variance with earlier studies on the intrinsic strangeness in the proton based on the $K^{+} \Lambda^{0}$ configuration for the strange component $10,11,12,13]$, which leads to negative values for $\mu_{s}$. It should be instructive to investigate the consequences of the configurations with the $\bar{s}$ in the ground state on other processes relevant to the intrinsic strangeness, such as the NuTeV anomaly problem [21, 22].

Equally instructive would be a comparison of the role of the analogous configurations for the non-strange pentaquark components in nucleon and $N^{*}$ to that of conventional meson cloud configurations. Thus the observation of an excess $\bar{d}$ over $\bar{u}$ antiquarks in the proton [23], which has been described in terms of a $n \pi^{+}$configuration [24], might alternately be described in terms of an $[u d u d] \bar{d}$ component in the proton. While the $N^{*}(1440)$ may contain significant $N \pi$ and $\Delta \pi$ components, its unusual properties might also be described in terms of admixtures of colored quark cluster configurations.
A recent chiral quark model calculation for the $u u d d \bar{s}$ pentaquark system 25] suggests that the configuration with both uudd and $\bar{s}$ in their ground state gives lower energy than the $[31]_{X}[4]_{F S}$ configurations. It should be interesting to extend that calculation to the configuration with uudd in the ground state and the $\bar{s}$ in its $P$ - wave state to see if it gives a higher energy than the lowest $[31]_{X}[4]_{F S}$ state. If so, it might provide an explanation for why the pentaquark component of the proton is in the lowest $[31]_{x}[4]_{F S}$ state. The penta-quark model with the most favorable configurations of the flavor-spin interaction with one quark in the $P$-state 26 naturally leads to a positive value for $\mu_{s}$.

In summary, the empirical indications for a positive strangeness magnetic moment of the proton suggest that the $s \bar{s}$ configuration in the proton is such that the $\bar{s}$ is the ground state and the uuds system in the $P$-state. This suggests that the $q q q q \bar{q}$ components in baryons may be mainly in colored quark cluster configurations rather than in "meson cloud" configurations. In the configuration with the lowest energy the positive empirical strangeness magnetic moment gives a direct estimate of the probability of the $s \bar{s}$ configuration.

\section{Acknowledgments}

B. S. Zou acknowledges the hospitality of the Helsinki Institute of Physics during course of this work. D. O. Riska acknowledges the hospitality of the W. K. Radiation laboratory, California Institute of Technology. Research supported in part by the Academy of Finland grant number 54038 and the National Natural Science Foundation of China.
[1] D. T. Spayde et al., Phys. Lett. B583, 79 (2004)

[2] K. Aniol et al., Phys. Rev. C69, 065501 (2004)

[3] F. Maas et al., nucl-ex/0412030

[4] H. Weigel et al., Phys. Lett. B353, 20 (1995)

[5] P. Geiger and N. Isgur, Phys. Rev. D55, 299 (1997)

[6] S.-T. Hong, B.-Y. Park and D. P. Min, Phys. Lett. B414, 229 (1997)

[7] V. Lyubovitskij et al., Phys. Rev. C66, 055204 (2002)

[8] R. Lewis, W. Wilcox and R. M. Woloshyn., Phys. Rev. D67, 013003 (2003)

[9] A. Silva, H. C. Kim and K. Goeke, Eur. Phys. A22, 481 (2004)

[10] M. Musolf and M. Burkhardt, Z. Phys. C61, 433 (1984)

[11] H. Forkel, F. S. Navarra and M. Nielsen, Phys. Rev. C61, 055206 (2000)

[12] L. Hannelius and D. O. Riska, Phys. Rev. C62 045204 (2000)

[13] X.S. Chen et al., Phys. Rev. C70, 015201 (2004)

[14] R. Jaffe and F. Wilczek, Phys. Rev. Lett. 91, 232003 (2003)

[15] E. Shuryak and I. Zahed, Phys. Lett. B589, 21 (2004)

[16] M. Karliner and H. Lipkin, Phys. Lett. B75, 249 (2003)
[17] C. Helminen and D. O. Riska, Nucl. Phys. A699, 624 (2002)

[18] L. Ya. Glozman and D. O. Riska, Phys. Rep. 268, 263 (1996)

[19] J.-Q. Chen, Group Representation Theory for Physicists, World Scientific, Singapore (1989)

[20] Y.R. Liu et al., Phys. Rev. C69, 035205 (2004)

[21] G.P. Zeller et al., Phys. Rev. Lett. 88091802 (2002)

[22] Y. Ding, R.G. Xu and B.Q. Ma, Phys. Lett. B607, 101 (2005); J. Alwall and G. Ingelman, Phys. Rev. D70, 111505(R) (2004); F.G. Cao and A.I. Signal, Phys. Lett. B559, 229 (2003)

[23] G.T. Garvey and J.C. Peng, Prog. Part. Nucl. Phys. 47, 203 (2001), and references therein.

[24] J. Speth and A.W. Thomas, Adv. Nucl. Phys. 24, 93 (1997)

[25] F. Huang et al., Phys. Lett. B586, 69 (2004)

[26] Fl. Stancu and D. O. Riska, Phys. Lett. B575 (2003)242, Fl. Stancu, Phys. Lett. B595 (2004)269, (erratum Phys. Lett. B598 (2004)295. 